Research Article

\title{
Evaluating Construction Risks of Modified Shield Machine Applicable to Soft Soils Based on Fuzzy Comprehensive Evaluation Method
}

\author{
Qingfei Luo $\mathbb{D}^{1},{ }^{1}$ Wei Li $\mathbb{D}^{2},{ }^{2}$ Honglin Su $\mathbb{D}^{3},{ }^{3}$ and Xinru Chen $\mathbb{D D}^{4}$ \\ ${ }^{1}$ School of Railway Transportation, Shanghai Institute of Technology, Shanghai 201499, China \\ ${ }^{2}$ School of Civil Engineering, Putian University, Putian 351100, Fujian, China \\ ${ }^{3}$ Sinohydro Engineering Bureau 7 Co., Ltd., Chengdu 610500, Sichuan, China \\ ${ }^{4}$ Shanghai Baoye Group Corp. Ltd., Shanghai 201100, China \\ Correspondence should be addressed to Wei Li; weiliyy@126.com
}

Received 15 September 2020; Revised 26 September 2020; Accepted 30 September 2020; Published 28 October 2020

Academic Editor: Zheng-zheng Wang

Copyright (C) 2020 Qingfei Luo et al. This is an open access article distributed under the Creative Commons Attribution License, which permits unrestricted use, distribution, and reproduction in any medium, provided the original work is properly cited.

\begin{abstract}
Metro tunnel sections in China can generally be divided into two types, those in granite formations $(D=6.0 \mathrm{~m})$ and those in soft soils $(D=6.2 \mathrm{~m})$, to which the same shield tunnel machine cannot be applied. The consequent low rate of machine utilization needs to be addressed. One solution is to modify shield machines which tend to tunnel granite strata with varying degrees of weathering $(D=6.0 \mathrm{~m})$ into those applicable in soft soils $(D=6.2 \mathrm{~m})$. Shield tunneling is a complex operation accompanied by potential risks, and accordingly it is demanded in risk evaluation and management. Hence, according to the construction features of modified shield machines in soft soil areas, this paper identifies relevant risks before establishing a specific model of risk evaluation by virtue of a fuzzy comprehensive evaluation method. This model weighs risk factors by triangular fuzzy numbers, and the membership function included is of L-R type that is frequently used in engineering. This risk evaluation model is applied to one section tunnel (Binhai New Town-Lianhua) of Metro Line 6 in Fuzhou City. Tunneling tests in the field uncover problems of the modified shield machine, including inappropriate tunneling parameters, segment dislocation, segment damage, and inadequate grouting. The result conforms to that produced by the risk evaluation model, which in turn proves the reliability of this model. Field data are also analyzed to address existing problems and to determine the appropriate tunneling parameters. The validity of these tunneling parameters is verified when surface settlement is measured.
\end{abstract}

\section{Introduction}

Metro tunnels hold different geology and operation speeds, thereby different cross-sections. Tunnel sections in China have two different boundary lines of construction: one is those located in soft soil areas $(D=6.2 \mathrm{~m})$, as represented by Shanghai; the other is those in granite strata with varying degrees of weathering $(D=6.0 \mathrm{~m})$, and a typical example is Shenzhen. The construction of tunnel sections with varying geology and diameters cannot utilize the same shield machine. This would pose a great challenge to the budget control of companies which are to construct both types. To enable a single shield machine to operate in geologically different strata, there are two methods. The first is to add materials to change the nature of soil, so that a single machine can be consistently employed $[1,2]$. The second is that the machine can be conditioned to the strata under construction by adjusting its cutterhead and screw conveyor [3-8]. With respect to the second method, Li and Yuan [9] transformed the cutterhead and other components of a slurry pressure balanced shield machine three times to enable it to tunnel various soil layers. Another example is that Zhang et al. [10] aimed to design a new type of shield machine which can successfully tunnel water-rich mylonite faults. This machine was designed to operate like an EPB in strata which were not full of water, and in water-rich layers, it was equipped with a specific system to remove slurry. The researches cited above focus on what adjustments can be 
made to ensure that a single shield machine can handle geologically different strata within the same tunnel section, while the machine's diameter remains unchanged. Few studies have been conducted to find out how to modify a shield machine so that it can be employed in tunnels which vary in geology and diameter. Research of this kind, however, is of vital importance, as it can further reduce the budget cost of construction and increase the rate of machine utilization.

Shield tunneling is complicated in operation and has many potential risks in which risk factors are of high degrees of fuzziness [11]. The adaptive modification adds new construction risks to the existing ones a shield machine has to face. Metros tend to be built in populous areas, implying that an accident can bring about heavy losses. Risk evaluation of shield tunneling construction usually adopts methods like machine learning $[12,13]$, neural network $[14,15]$, and fuzzy comprehensive evaluation [16-18]. The third method, by virtue of its high accuracy, practicality, and easiness, is widely used in most industries including finance $[19,20]$, materials [21], environmental protection [22], and electricity [23]. The fuzzy comprehensive evaluation method is employed to evaluate the risk of shield tunneling, as well as other aspects of engineering [24-26], for example, assessing road conditions $[27,28]$, calculating the durability of reinforced concrete structures [29], and evaluating the safety of deep foundation pits of metro stations [30]. The paper mentioned before mainly used AHP to weigh risk factors. AHP utilizes the traditional scale table, of which the scales of values are derived from pairwise comparisons and are integrated into a risk matrix. After the consistency check, the maximum eigenvalue of the matrix is regarded as the weight vector of the risk. Yet, this method is too subjective, for the consistency check depends on the researcher's experience, and the lack of theoretical foundation cannot guarantee its precision $[31,32]$. The weight of each risk represents its own significance, so the weighting process must be scientific; if not, the final assessment will be affected. To avoid subjectivity, the matrix is optimized by triangular fuzzy numbers [33]. Compared with traditional risk weighting, triangular fuzzy numbers can give the upper and lower limits of a risk, which can lower the researcher's subjectivity to some extent.

During the risk evaluation of shield tunneling construction, the fuzzy comprehensive evaluation is mainly used to monitor tunnel conditions [34], decide the type of shield machines [35, 36], and assess the risk of water inrush during tunnel construction [37, 38], among others. Less consideration is given to the construction risk of modified shield machines applicable to soft soils, its identification, and the corresponding evaluation model. This should not be the case, however. The identification and evaluation model of this kind of risk should be emphasized. Apart from that, tunneling tests on site are needed to examine if the modified shield machine can reach the original expectation, especially when it is applied to a tunnel section which is diametrically different from the previous one in geology. Problems need to be identified directly in the process. After the test, relevant solutions and the resetting of tunneling parameters should be considered.
Based on one section (Binhai New Town-Lianhua) of Metro Line 6 in Fuzhou City, this paper discusses how composite EPB shield machines which tend to tunnel granite strata $(D=6 \mathrm{~m})$ can be modified and applied to soft soils $(D=6.2 \mathrm{~m})$. After identifying the construction risk of the modified shield machine in soft soils, this paper weighs risk factors by triangular fuzzy numbers and calculates the membership grades of risk events by L-R membership function, so as to provide a fuzzy comprehensive evaluation model which is tailored to the application of the modified shield machine. This model is used to rate the risk of this project. Tunneling tests on-site show that problems like inappropriate tunneling parameters segment dislocation and damage can occur when the modified shield machine is working at soft soils. This result is in line with that produced by the evaluation model, proving the reliability of this model. These problems are carefully considered before figuring out appropriate tunneling parameters and possible solutions. With the assistance of surface settlement measurement, these new parameters are certified as right.

\section{Risk Identification}

Shield tunneling in soft soils often faces risks from the following five aspects: shield launching and receiving, shield tunneling, segment assembly, grouting, and the working environment [39]. Shield machines modified to soft soils are likely to be at risk in shield tunneling, segment assembly, and grouting, for the reason that they might not undergo an overall modification. Risks of the three aspects will be further introduced.

2.1. Risks of Shield Tunneling. Shield construction in soft soils might be risky because of excessive rotation of the shield machine, deviation from the designed tunneling axis, inappropriate tunneling parameters, blockage in earth conveying, and leakage in the shield sealing system. The latter three problems can easily happen when the modified shield machine is tunneling soft soils.

Tunneling parameters include total shield thrust, cutterhead torque and rotation speed, tunneling speed, earth pressure, and excavation volume. These parameters vary with the geological conditions of strata; for example, earth pressure is decided by soil bulk density, and the torque and rotation speed of cutterhead vary from stratum to stratum. If a shield machine is modified and applied to a stratum different from the previous one, problems caused by inappropriate tunneling parameters are likely to occur during construction.

The shield machine's internal structure needs to be modified before it can be applied to soft soils. Modification in the internal structure can cause misalignment between the screw and the shell. Tunneling in soft soils requires screw conveyors of higher power than in solid rocks; hence the previous screw machine may not be powerful enough to convey earth smoothly in soft soils.

Leakage in the shield sealing system is caused when the shield tail gap is so large that sealing devices fail to function 
well. This excessively large gap occurs if the sealing connection is not elastic enough or the shield tail is not aligned with the segment. The shield tail gap can also be enlarged when the machine has been used for a long time, which is usually the case of the modified shield machine. All these are possible reasons for the reduced tightness.

2.2. Risks of Segment Assembly. Segment-related risks during the tunneling construction in soft soils include segment dislocation, damage, and attitude deviation. Dislocation of segments has three main causes: (1) nonstandard segment assembly and the consequent loose bolted connection can make segments dislocated; (2) changes of shield tail gap in shield deviation correction can dislocate segments; (3) when a shield is tunneling through the shallow-covered soil section in silt strata, its attitude is difficult to be controlled and it tends to advance at a high speed. Under such circumstances, the axes of the shield and the segment are prone to diverge, leading to the dislocation of segments.

Segment damage might happen for the following three reasons: (1) if the center of the segment is not in line with that of the shield machine, there will be an uneven force on the segment. The lateral pressure on it will exceed its designed value of tensile or compressive strength and cause damage; (2) when the segment is transported and stacked, some bumps would result in cracks on it; (3) the segment might be broken by the uneven force exerted during the process of axis deviation correction.

Segment attitude deviation can be caused (1) if the initial setting time of synchronous grouting is too long and the grouting materials surrounding the segment cannot bind them immediately and (2) if, in a shallow tunnel, the pressure of the abundant groundwater is more than the segment can bear and results in segment uplifting.

Briefly, the above risks mainly occur in the procedures of segment transportation, segment assembly, shield deviation correction, and grouting. If a shield machine is to be conditioned to the tunneling construction in soft soils, its thrust system, cutterhead and cutting tool, and shield need to be modified considerably. If the modification is incomplete, the safety during construction cannot be guaranteed, particularly in the steps mentioned before. This is why segment assembly is of high risk for the modified shield machines applicable to soft soils.

2.3. Risks of Grouting. Grouting-related problems in soft soils tunneling can occur if the grout is not injected in a timely and consistent way, the pipes are blocked, or the grout is poor in quality.

Like shield tunneling and segment assembling, groutingrelated factors also vary with the geology of strata. The modified shield machine requires different grouting material, grouting volume, and grouting pressure when the strata under construction are shifted from rocks to soft soils. If these factors are not thoroughly considered, grouting-related risks might be brought about. These risks can be intensified if the new grout is mixed with the residue accumulated in grouting pipes. The residue is grouting materials which had been applied to a stratum of totally different geological conditions. Such a mixture will give rise to more blockages in the grouting pipes. Therefore groutingrelated risks are of significance during the operation of the modified shield machine applicable to soft soils.

\section{Fuzzy Comprehensive Evaluation Model}

3.1. Establishing the Risk Evaluation System. The risk index system for modified shield construction applicable to soft soils is set up by the analytic hierarchy process (AHP) according to the identification results of the above construction risk factors. The risk index system can be roughly divided into three layers, which are, respectively, the target layer, the first-level index layer, and the second-level index layer, among which the target layer is the first layer that represents the final evaluation targets and is recorded as $\mathrm{U}$, namely, the safety risk evaluation for modified shield machine construction applicable to soft soils. The second and third layers are, respectively, the first-level index layer (risk accidents) and the second-level index layer (potential risk factors), among which the first-level index layer should be denoted as $U_{i}(i=1,2,3,4,5)$, while the second-level index layer is denoted as $U_{i i}$. Please see Figure 1.

The risks of modified shield machine construction suitable for soft soils are divided into 5 levels: "extremely low risk," "low risk," "medium risk," "high risk," and "extremely high risk," which are denoted as $V$ :

$$
V=\left\{v_{1}, v_{2}, v_{3}, v_{4}, v_{5}\right\} .
$$

\subsection{Determining Risk Weights}

3.2.1. Building up Triangular Fuzzy Number Complementary Judgment Matrix. Experts conduct paired comparison on the importance of risks at the same level by referring to the fuzzy scale table (see Table 1) and the risk evaluation system, to obtain the triangular fuzzy number complementary matrix $A$ :

$$
\left\{\begin{array}{l}
A=\left(\tilde{a}_{\mathrm{ij}}\right)_{m \times n}, \\
\tilde{a}_{\mathrm{ij}}=\left(l_{\mathrm{ij}}, m_{\mathrm{ij}}, u_{\mathrm{ij}}\right),
\end{array}\right.
$$

where $\tilde{a}_{\mathrm{ij}}$ refers to the ratio of the relative importance of the No. $i$ factor to No. $j$ factor, and $l_{\mathrm{ij}}, m_{\mathrm{ij}}$, and $u_{\mathrm{ij}}$, respectively, refer to the lower bound value, most probable value, and upper bound value in experts' judgment on risks. Meanwhile, these three also satisfy the conditions $l_{\mathrm{ij}}<m_{\mathrm{ij}}<u_{\mathrm{ij}}$, $l_{\mathrm{ij}}+u_{\mathrm{ij}}=1$, and $u_{\mathrm{ij}}+l_{\mathrm{ij}}=1$. And the value of $\left|u_{\mathrm{ij}}-l_{\mathrm{ij}}\right|$ indicates the fuzzy degree of this judgment interval. When the value is 0 , it means that the judgment is not fuzzy; instead, it is an accurate judgment.

3.2.2. Determining Initial Fuzzy Weights. The initial weight of risk represents the relative importance ranking of risk events at the same level to the superior level. Supposing that there are a total of $n$ indices in a certain second-level index 


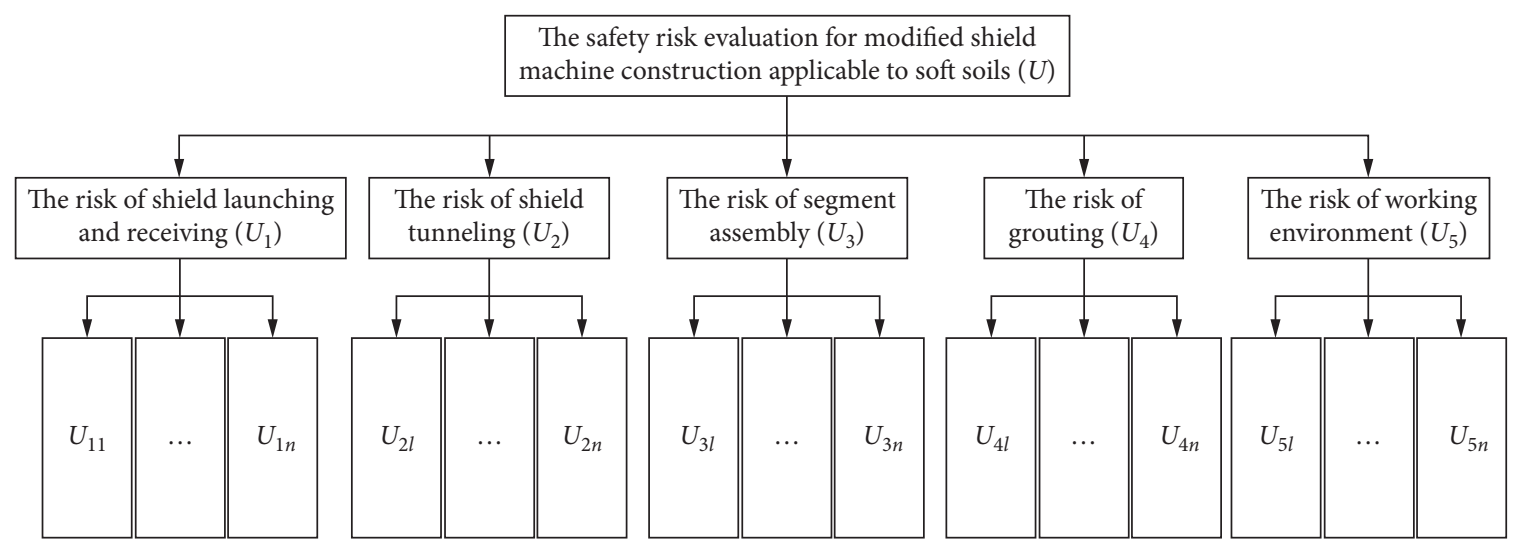

Figure 1: Risk evaluation system.

TABLE 1: Fuzzy scale table.

\begin{tabular}{lc}
\hline Scale & Meaning \\
\hline 0.1 & Risk $i$ is absolutely not as important as risk $j$ \\
0.3 & Risk $i$ is obviously not as important as risk $j$ \\
0.5 & Risk $i$ has the same importance as risk $j$ \\
0.7 & Risk $i$ is obviously more important than risk $j$ \\
0.9 & Risk $i$ is absolutely more important than risk $j$ \\
\hline
\end{tabular}

where $0.2,0.4,0.6$, and 0.8 are the median of adjacent judgment intervals.

layer, the corresponding initial fuzzy weight $\widetilde{w}_{i}$ of No. $i$ index could be obtained by the following calculation:

$$
\left\{\begin{array}{l}
\widetilde{w}_{i}=\frac{\sum_{j=1}^{n} a_{\mathrm{ij}}}{\sum_{i=1}^{n} \sum_{j=1}^{n} a_{\mathrm{ij}}}, \quad i=1,2, \ldots, n, \\
\frac{\sum_{j=1}^{n} a_{\mathrm{ij}}}{\sum_{i=1}^{n} \sum_{j=1}^{n} a_{\mathrm{ij}}}=\left(\frac{\sum_{j=1}^{n} l_{\mathrm{ij}}}{\sum_{i=1}^{n} \sum_{j=1}^{n} u_{\mathrm{ij}}}, \frac{\sum_{j=1}^{n} m_{\mathrm{ij}}}{\sum_{i=1}^{n} \sum_{j=1}^{n} m_{\mathrm{ij}}}, \frac{\sum_{j=1}^{n} u_{\mathrm{ij}}}{\sum_{i=1}^{n} \sum_{j=1}^{n} l_{\mathrm{ij}}}\right) .
\end{array}\right.
$$

3.2.3. Calculating Weight Vectors. First, the possibility that the vectors of the initial fuzzy weights at the same level are $\widetilde{w}_{1}>\widetilde{w}_{2}$ should be calculated, namely, $P\left(\widetilde{w}_{1}>\widetilde{w}_{2}\right)$. It can be calculated by the following formula:

$$
P\left(\widetilde{w}_{1} \geq \widetilde{w}_{2}\right)= \begin{cases}1, & m_{1} \geq m_{2}, \\ \frac{l_{2}-u_{1}}{\left(m_{1}-u_{1}\right)-\left(m_{2}-l_{2}\right)}, & m_{1}<m_{2}, u_{1} \geq l_{2}, \\ 0, & \text { otherwise. }\end{cases}
$$

Next, the possibility that $\widetilde{w}_{1}$ is greater than or equal to the vector of the other fuzzy weight at the same level should be considered, namely, $P\left(\widetilde{w}_{1} \geq \widetilde{w}_{2}, \widetilde{w}_{3}, \ldots, \widetilde{w}_{n}\right)$. It can be calculated by the following formula:

$$
P\left(\widetilde{w}_{1} \geq \widetilde{w}_{2}, \widetilde{w}_{3}, \ldots, \widetilde{w}_{n}\right)=\min P\left(\widetilde{w}_{1} \geq \widetilde{w}_{i}\right), \quad i=2, \ldots, n .
$$

Set $d^{\prime}\left(A_{i}\right)=\min P\left(\widetilde{w}_{i} \geq \widetilde{w}_{k}\right), \quad k=1,2, \ldots, n$, and $k \neq i$. Then, the weight vector $W^{\prime}$ of this hierarchy should be

$$
W^{\prime}=\left(d^{\prime}\left(A_{1}\right), d^{\prime}\left(A_{2}\right), \ldots, d^{\prime}\left(A_{n}\right)\right) \text {. }
$$

After being normalized, the final weight vector $W$ could be obtained as follows:

$$
W=\left(\frac{d^{\prime}\left(A_{1}\right)}{\sum_{i=1}^{n} d^{\prime}\left(A_{i}\right)}, \frac{d^{\prime}\left(A_{2}\right)}{\sum_{i=1}^{n} d^{\prime}\left(A_{i}\right)}, \ldots, \frac{d^{\prime}\left(A_{n}\right)}{\sum_{i=1}^{n} d^{\prime}\left(A_{i}\right)}\right) .
$$

3.3. Calculating Membership Degree. First, the consequence valuation of risk events $(C)$ and the probability valuation of risk occurrence $(P)$ are conducted by scoring of experts. And the valuing methods for the above two are as shown in Tables 2 and 3.

After that, the specific form of the membership function is determined. The membership function is calculated by L-R function; see the following formula:

$$
r_{\mathrm{ij}}(x)= \begin{cases}0, & x \leq a \text { or } x \geq b, \\ L(x), & a<x<m, \\ R(x), & m \leq x<b,\end{cases}
$$

where $a$ and $b$ are both positive numbers. For specific details, please see $(9) \sim(13)$.

$$
\begin{aligned}
& \text { Extremely low risk : } r_{i 1}= \begin{cases}1, & 0<x \leq 3, \\
4-x, & 3<x \leq 4, \\
0, & x>4,\end{cases} \\
& \text { low risk }: r_{i 2}= \begin{cases}x-3, & 3 \leq x<4, \\
1, & 4 \leq x \leq 7, \\
8-x, & 7<x \leq 8, \\
0, & x<3 \text { or } x>8,\end{cases} \\
& \text { medium risk }: r_{i 3}= \begin{cases}x-7, & 7 \leq x<8, \\
1, & 8 \leq x \leq 15, \\
16-x, & 15<x \leq 16, \\
0, & x<7 \text { or } x>16,\end{cases}
\end{aligned}
$$


TABLE 2: Consequence of valuation of risk events.

\begin{tabular}{lcc}
\hline Degree & Valuation & Description \\
\hline Slight & 1 & Does not result in significant loss \\
Medium & 2 & Results in a few losses \\
Severe & 3 & Results in compensable losses \\
Material & 4 & Results in significant but compensable \\
Disastrous & 5 & Results in uncompensable losses \\
\hline
\end{tabular}

where $1.5,2.5,3.5$, and 4.5 indicate that the severity of the risk event is between the adjacent degrees.

TABLE 3: Probability valuation of risk occurrence.

\begin{tabular}{lcc}
\hline Degree & Valuation & Description \\
\hline Rare & 1 & The risk is rarely seen \\
Occasional & 2 & The risk is unlikely to happen \\
Possible & 3 & The risk may happen \\
Anticipated & 4 & The risk may happen repeatedly \\
Frequent & 5 & The risk may happen frequently \\
\hline
\end{tabular}

where $1.5,2.5,3.5$, and 4.5 indicate that the probability of occurrence of the risk event is between the adjacent levels.

$$
\begin{aligned}
& \text { high risk }: r_{i 4}= \begin{cases}x-15, & 15 \leq x<16, \\
1, & 16 \leq x \leq 20, \\
16-x, & 20<x \leq 21, \\
0, & x<15 \text { or } x>21,\end{cases} \\
& \text { extremely high risk }: r_{i 5}= \begin{cases}x-20, & 20 \leq x<21, \\
1, & 21 \leq x \leq 25, \\
0, & x<20 .\end{cases}
\end{aligned}
$$

Finally, the product of the consequence valuation of the risk event and the probability valuation of the risk occurrence is, respectively, brought into the above five membership functions, and then the membership degrees of the risk events to the five risk levels can be obtained. The membership degrees of the risk events at the same level are then composed into judgment matrix $R_{i}(i=1,2, \ldots, 5)$ :

$$
R_{i}=\left(\begin{array}{ccc}
r_{11} & \cdots & r_{51} \\
\vdots & \ddots & \vdots \\
r_{n 1} & \cdots & r_{5 n}
\end{array}\right)_{n \times 5},
$$

where $n$ refers to the specific number of indices of a certain second-level index layer.

3.4. Fuzzy Comprehensive Judgment. First, calculate the comprehensive judgment matrix of the first-level index layer $B_{i}$ :

$$
B_{i}=W_{i} \circ R_{i}=\left[b_{i 1}, b_{i 2}, b_{i 3}, b_{i 4}, b_{i 5}\right], \quad i=1,2, \ldots, 5,
$$

where $W_{i}$ refers to the weight of risk event in the first-level index layer and $\circ$ refers to the fuzzy operator.

After that, the comprehensive judgment matrices of all first-level index layers are compounded into the judgment matrix $R$ of the target index layer:

$$
R=\left[B_{1}, B_{2}, B_{3}, B_{4}, B_{5}\right]^{T} .
$$

Finally, calculate the comprehensive judgment matrix $B$ of the target layer as follows:

$$
B=W \circ R=\left[b_{1}, b_{2}, b_{3}, b_{4}, b_{5}\right],
$$

where $W$ refers to the weight vector of the target layer.

According to the maximum membership principle, evaluation index $v_{i}$ that the maximum element of $B$ corresponds to is taken, namely, the construction risk level of the modified shield machine suitable for soft soil.

\section{Case Study}

\subsection{Project Overview}

4.1.1. Hydrological and Geological Conditions. The metro section between Binhai New Town Station and Lianhua Station is $1190 \mathrm{~m}$ long, and the right tunnel is $14 \mathrm{~m}$ away from the left one. The longitudinal profile of this tunnel section is a V-shaped slope. This section has a connection passage and pumping station at XK27 +800.933 . The minimum thickness of overburden layer along the line is $6.81 \mathrm{~m}$ at Lianhua Station, and the maximum depth is $14.51 \mathrm{~m}$ at the connection passage. The thickness of Binhai New Town Station is $7.36 \mathrm{~m}$. The surface in this section is mainly covered by farms and ponds, and there are no large buildings and underground utilities within the construction area. This tunnel section largely passes through strata with soft soils (silt or mucky soil), fine-medium sand with mud, and sandy clay, as shown in Figure 2.

The surface is scattered with a few small rivers which are $10-30 \mathrm{~m}$ wide and $1.00-4.00 \mathrm{~m}$ deep. These rivers are not equipped with impervious facilities and are not sensitive to tides. The groundwater level is around $0.00-1.89 \mathrm{~m}$, with an annual change between 1.0 and $1.5 \mathrm{~m}$. This section is tunneled by the modified shield machine applicable in soft soils. The shield machine $(D=6.28 \mathrm{~m})$ is originally applied to the upper-soft lower-hard ground between Taoyuancun Station and Shenyun Station in Shenzhen Metro Line 7. After modification, its diameter is now $6.48 \mathrm{~m}$ and can be utilized to tunnel soft ground. In this project, the shield machine is launched at Binhai New Town Station and received at Lianhua Station, and the construction organization is illustrated in Figure 3.

4.1.2. Modifications of Shield Machines Conditioned to Soft Soils. Considering the tunnel diameter and the geology of the soft ground between Binhai New Town Station and Lianhua Station in Fuzhou Metro Line 6, the shield machine has undergone the following modifications:

(1) Enlarging Shield Outer Diameter. The outer diameter of the shield is enlarged by $0.2 \mathrm{~m}$, from $6.28 \mathrm{~m}$ to $6.48 \mathrm{~m}$. This enlargement will increase the perimeter of the shield (front shield, middle shield, and shield tail). The shield is easy to be worn out in the uppersoft lower-hard ground Figure 4(a), so a new one is applied to the modified machine Figure 4(b). Yet, the internal system remains unchanged, including the articulation seal, emergency airbag, tail brush, grout 


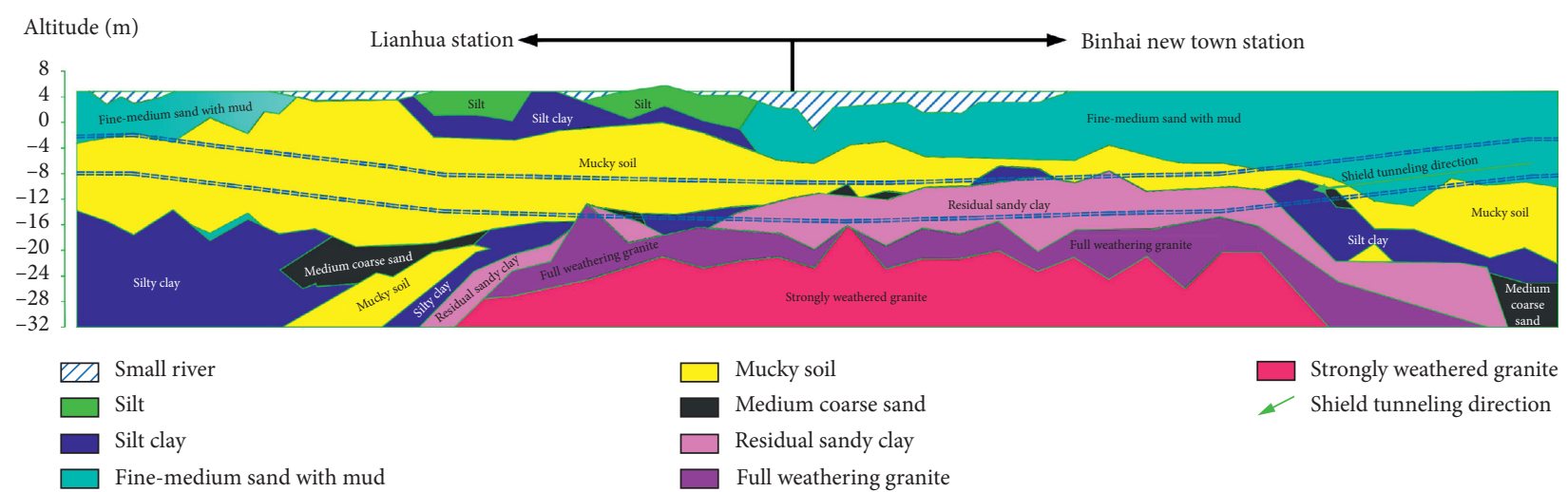

FIgUre 2: Longitudinal geological profile of Binhai New Town-Lianhua.

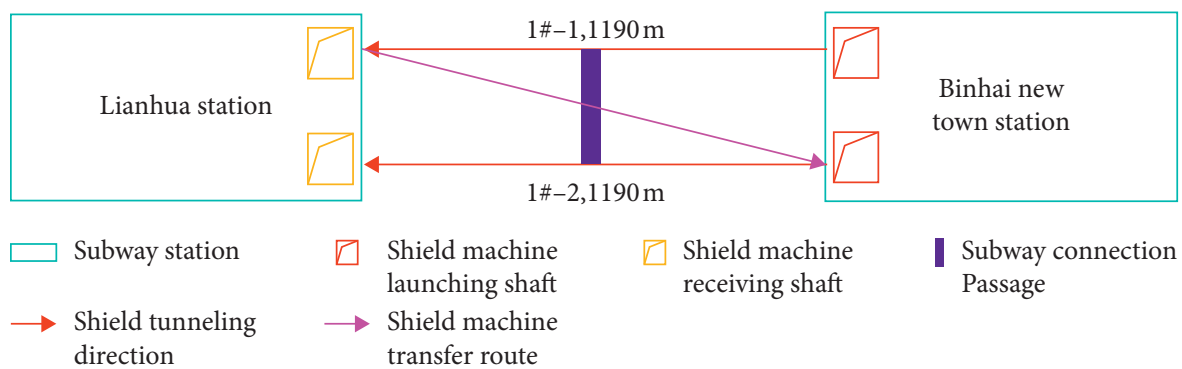

Figure 3: Construction organization diagram of Binhai New Town-Lianhua.

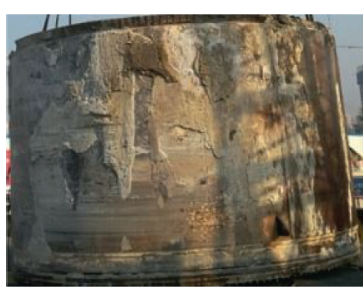

(a)

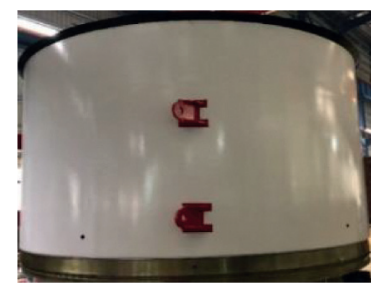

(b)

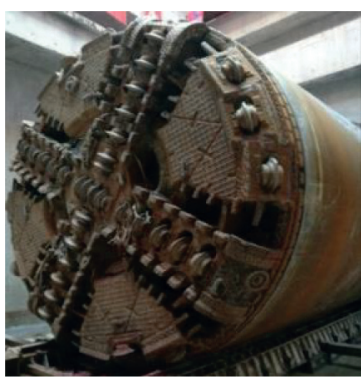

(c)

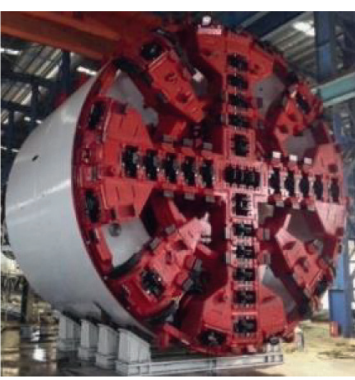

(d)

Figure 4: Unmodified and modified shield machines. (a) Unmodified shield. (b) Modified shield with enlarged diameter. (c) Cutterhead in upper-soft lower-hard ground. (d) Cutterhead in soft soil.

cover, clamping pad, trailer pad, screw conveyor, segment erector, belt conveyor, circulating water system, guiding system, hydraulic system, display screen, boring control box, internal control line of the machine, and two articulation cylinders.

(2) Changing Cutterhead and Tools. In the upper-soft lower-hard ground where the strength of rock is very high, the cutterhead is easy to be worn, and the cutter tools are generally hobs. The machine in the beginning is equipped with 4 central double-edge hobs and 31 single-edge hobs Figure 4(c), together with 52 scrapers, 8 edge blades, and 1 super digging knife. The aperture of the cutterhead is $30 \%$. In contrast, the soft soil between Binhai New Town Station and Lianhua Station requires that the main cutter tool is the tearing knife, including 8 main tearing knives in reserve, 32 ordinary tearing knives, and 23 welding tearing knives. Apart from that, the modified shield machine has 16 side scrapers, 36 cutters, 1 super digging knife, 16 big ring protection knives, and 8 gauge knives. The cutter-head aperture is increased to $40 \%$ Figure $4(\mathrm{~d})$. The cutterhead is driven by a hydraulic system with a power of $945 \mathrm{~kW}$. The rated torque of the cutterhead is $6228 \mathrm{kN} \cdot \mathrm{m}$, and its maximum release torque stands at $7440 \mathrm{kN} \cdot \mathrm{m}$. The maximum rotating speed can reach $4.4 \mathrm{rpm}$ and it contains 6 foam injection holes.

(3) Modifying the Thrust System. The thrust system of the unmodified shield machine has 30 thrust oil cylinders. The modified shield machine has a larger outer diameter, and accordingly it is equipped with 2 more oil cylinders, 32 in total. The previous thrust 
cylinders consist of 10 sets of double cylinders and 10 sets of single cylinders, with 5 sets at the left, the right, the top, and the bottom, respectively. The modified thrust system holds 16 sets of double cylinders, with 3 sets at the top, 5 at the bottom, 4 at the right, and 4 at the left. The model of the oil cylinder is $\varnothing 220 / 180 \times 2200 \mathrm{~mm}$, and the thrust power it can provide is $1330 \mathrm{kN}$. The total thrust power of the unmodified machine reaches $39900 \mathrm{kN}$, and that of the modified machine $42560 \mathrm{kN}$. The thrust stroke of the cylinder is $2200 \mathrm{~mm}$, and its speed arrives at $80 \mathrm{~mm} / \mathrm{min}$. There are 4 cylinders which wear stroke sensors.

4.2. Setting up the Risk Evaluation System. The risk evaluation system (see Table 4) for this project is set up by the expert group based on discussion and review of a large number of references, combined with the actual situation of the project. This system is composed of 5 first-level indices and 20 second-level indices.

Besides, the above-mentioned evaluation set is taken for this project, including "extremely low risk," "low risk," "medium risk," "high risk," and "extremely high risk," which are denoted as $V=\left\{v_{1}, v_{2}, v_{3}, v_{4}, v_{5}\right\}$.

4.3. Application of the Fuzzy Comprehensive Evaluation Model. By taking the shield launching and receiving risk $U_{1}$ for instance, it is hereby to introduce how to use this model.

4.3.1. Determining Risk Weight Vector. First of all, the complementary matrix $A_{U_{1}}$ of triangular fuzzy numbers on shield launching and receiving risks is obtained by expert scoring:

$$
A_{U_{1}}=\left(\begin{array}{llllll}
(0.50,0.50,0.50) & (0.20,0.32,0.44) & (0.28,0.43,0.48) & (0.25,0.36,0.46) & (0.31,0.45,0.49) \\
(0.56,0.68,0.80) & (0.50,0.50,0.50) & (0.53,0.59,0.74) & (0.51,0.55,0.69) & (0.54,0.64,0.79) \\
(0.52,0.57,0.72) & (0.26,0.41,0.47) & (0.50,0.50,0.50) & (0.27,0.45,0.48) & (0.51,0.55,0.71) \\
(0.54,0.64,0.75) & (0.31,0.45,0.49) & (0.52,0.55,0.73) & (0.50,0.50,0.50) & (0.53,0.58,0.74) \\
(0.51,0.55,0.69) & (0.21,0.36,0.46) & (0.29,0.45,0.49) & (0.26,0.42,0.47) & (0.50,0.50,0.50)
\end{array}\right) .
$$

Calculate the initial fuzzy weight of each factor under the risk of shield launching and receiving according to (3) as follows:

$$
\left\{\begin{array}{l}
\widetilde{w}_{U_{11}}=\left(\begin{array}{lll}
0.106 & 0.165 & 0.228
\end{array}\right) \\
\widetilde{w}_{U_{12}}=\left(\begin{array}{lll}
0.181 & 0.237 & 0.338
\end{array}\right) \\
\widetilde{w}_{U_{13}}=\left(\begin{array}{lll}
0.141 & 0.198 & 0.277
\end{array}\right) \\
\widetilde{w}_{U_{14}}=\left(\begin{array}{lll}
0.165 & 0.218 & 0.308
\end{array}\right) \\
\widetilde{w}_{U_{15}}=\left(\begin{array}{lll}
0.121 & 0.182 & 0.251
\end{array}\right)
\end{array}\right.
$$

The weight vector $W_{U_{1}}^{\prime}$ of each factor under the shield launching and receiving risk can be calculated by (4) (5) as follows:

$$
W_{U_{1}}^{\prime}=\left(\begin{array}{lllll}
0.394 & 1 & 0.714 & 0.869 & 0.562
\end{array}\right) .
$$

The final weight vector $W_{U_{1}}$ under the risk of shield launching and receiving can be obtained by normalization by (7) as follows:

$$
W_{U_{1}}=\left(\begin{array}{lllll}
0.111 & 0.283 & 0.201 & 0.246 & 0.159
\end{array}\right) .
$$

The final weight vectors $W_{U_{2}} \sim W_{U_{5}}$ of the remaining first-level index layers are as follows:

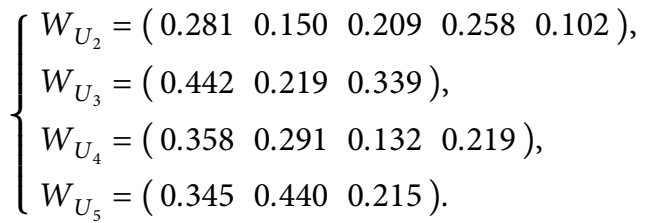

Final weight vector $W$ of the target layer is as follows:

$$
W=\left(\begin{array}{lllll}
0.211 & 0.274 & 0.122 & 0.240 & 0.153
\end{array}\right) .
$$

4.3.2. Determining Risk Membership. According to the valuing methods of Tables 2 and 3, the product of the consequence valuation $(C)$ and the occurrence probability valuation $(P)$ of risks under $U_{1}$ is determined by expert scoring, as shown in Table 5.

The judgment matrix $R_{U_{1}}$ for the shield launching and receiving risks can be obtained by bringing the aforementioned product into the membership function:

$$
R_{U_{1}}=\left(\begin{array}{ccccc}
0.25 & 0.75 & 0 & 0 & 0 \\
0 & 0 & 1 & 0 & 0 \\
0 & 1 & 0 & 0 & 0 \\
0 & 1 & 0 & 0 & 0 \\
0.25 & 0.75 & 0 & 0 & 0
\end{array}\right) .
$$

The results of the judgment matrix of the remaining firstlevel index layers $R_{U_{2}} \sim R_{U_{5}}$ are as follows:

$$
R_{U_{2}}=\left(\begin{array}{ccccc}
0 & 0 & 0 & 0.75 & 0.25 \\
0 & 0 & 1 & 0 & 0 \\
0 & 0 & 0 & 1 & 0 \\
0 & 0 & 0 & 1 & 0 \\
0 & 0 & 1 & 0 & 0
\end{array}\right),
$$


Table 4: The risk evaluation system of Binhai New Town station-Lianhua station of Metro Line 6 in Fuzhou City.

\begin{tabular}{|c|c|c|}
\hline The target layer & The first-level index layer & The second-level index layer \\
\hline $\begin{array}{l}\text { The safety risk evaluation } \\
\text { for modified shield machine } \\
\text { construction applicable } \\
\text { to soft soils }(U)\end{array}$ & The risk of shield tunneling $\left(U_{2}\right)$ & $\begin{array}{c}\text { Deformation of the base }\left(U_{11}\right) \\
\text { Deviation from the axis during launching }\left(U_{12}\right) \\
\text { Soil influx into receiving shaft after dismantling portal }\left(U_{13}\right) \\
\text { Deviation from the axis during receiving }\left(U_{14}\right) \\
\text { Deformation of support during receiving }\left(U_{15}\right) \\
\text { Inappropriate tunneling parameters }\left(U_{21}\right) \\
\text { Excessive rotation of the shield machine }\left(U_{22}\right) \\
\text { Blockage in earth conveying }\left(U_{23}\right) \\
\text { Deviation from the designed tunneling }\left(U_{24}\right) \\
\text { Leakage in the shield sealing system }\left(U_{25}\right) \\
\text { Segment dislocation }\left(U_{31}\right) \\
\text { Segment deviation }\left(U_{32}\right) \\
\text { Segment damage }\left(U_{33}\right) \\
\text { Not grouting in time }\left(U_{41}\right) \\
\text { Inadequate grouting }\left(U_{42}\right) \\
\text { Clogging of the grouting pipe }\left(U_{43}\right) \\
\text { Low quality of the grouting material }\left(U_{44}\right) \\
\text { Effect of groundwater }\left(U_{51}\right) \\
\text { The effect of tunnel underneath the river }\left(U_{52}\right) \\
\text { Tunneling through unfavorable geology }\left(U_{53}\right)\end{array}$ \\
\hline
\end{tabular}

TABLE 5: Products of the consequence valuation and the occurrence probability valuation of risks under $U_{1}$.

\begin{tabular}{lccccc}
\hline Risk index & $U_{11}$ & $U_{12}$ & $U_{13}$ & $U_{14}$ & $U_{15}$ \\
\hline$C$ & 2.50 & 3.00 & 2.50 & 2.75 & 2.50 \\
$P$ & 1.50 & 2.75 & 2.00 & 2.50 & 1.50 \\
$C \times P$ & 3.75 & 8.25 & 5.00 & 6.88 & 3.75 \\
\hline
\end{tabular}

$$
\begin{aligned}
R_{U_{3}} & =\left(\begin{array}{lllll}
0 & 0 & 0 & 1 & 0 \\
0 & 0 & 1 & 0 & 0 \\
0 & 0 & 0 & 1 & 0
\end{array}\right), \\
R_{U_{4}} & =\left(\begin{array}{lllll}
0 & 0 & 0 & 1 & 0 \\
0 & 0 & 0 & 1 & 0 \\
0 & 0 & 1 & 0 & 0 \\
0 & 0 & 1 & 0 & 0
\end{array}\right), \\
R_{U_{5}} & =\left(\begin{array}{ccccc}
0 & 1 & 0 & 0 & 0 \\
0 & 0.56 & 0.44 & 0 & 0 \\
0.25 & 0.75 & 0 & 0 & 0
\end{array}\right) .
\end{aligned}
$$

4.3.3. Fuzzy Comprehensive Judgment. Calculate the comprehensive judgment matrix $B_{U_{1}}$ of the shield launching and receiving risks by (15) as follows:

$$
B_{U_{1}}=\left(\begin{array}{lllll}
0.07 & 0.65 & 0.28 & 0 & 0
\end{array}\right) .
$$

The comprehensive judgment matrices $B_{U_{2}} \sim B_{U_{5}}$ of the remaining first-level index layers are as follows:

$$
\left\{\begin{array}{l}
B_{U_{2}}=\left(\begin{array}{lllll}
0 & 0 & 0.25 & 0.68 & 0.07
\end{array}\right), \\
B_{U_{3}}=\left(\begin{array}{lllll}
0 & 0 & 0.22 & 0.78 & 0
\end{array}\right), \\
B_{U_{4}}=\left(\begin{array}{lllll}
0 & 0 & 0.35 & 0.65 & 0
\end{array}\right), \\
B_{U_{5}}=\left(\begin{array}{lllll}
0.05 & 0.75 & 0.19 & 0 & 0
\end{array}\right) .
\end{array}\right.
$$

The judgment matrix $R$ of the target layer can be obtained by (16) as follows:

$$
R=\left(\begin{array}{ccccc}
0.07 & 0.65 & 0.28 & 0 & 0 \\
0 & 0 & 0.25 & 0.68 & 0.07 \\
0 & 0 & 0.22 & 0.78 & 0 \\
0 & 0 & 0.35 & 0.65 & 0 \\
0.05 & 0.75 & 0.19 & 0 & 0
\end{array}\right) .
$$

The comprehensive judgment matrix $B$ of the target layer can be obtained by (17) as follows:

$$
B=\left(\begin{array}{lllll}
0.02 & 0.25 & 0.27 & 0.44 & 0.02
\end{array}\right) .
$$

4.4. Risk Evaluation Results. According to the maximum membership principle, the project is finally evaluated as having high risk, among which the shield launching and receiving risk $U_{1}$ is evaluated as low risk, shield tunneling risk $U_{2}$ as high risk, segment assembly risk $U_{3}$ as high risk, grouting risk $U_{4}$ as high risk, and environmental risk $U_{5}$ as low risk.

It can be known from the above risk evaluation results that the risks of shield tunneling, segment assembly, and grouting should be paid great attention during the construction process of the modified shield machine applicable to soft soils. 
4.5. Verifying the Effectiveness of the Risk Evaluation. In the above case, the level that the maximum membership corresponds to is taken as the final grade of the evaluated object. Though it is easy to operate, it may lead to irrational conclusions in circumstances of similar membership degrees.

Therefore, it is necessary to verify the effectiveness of the final grade of the evaluated object. Set $\alpha$ as the effectiveness index; then, the calculation formula and the corresponding effectiveness interval are as follows [40]:

$$
\alpha=\frac{m \beta-1}{2 \gamma(m-1)},
$$

where $\beta$ refers to the maximum membership value in judgment matrix $B$ of the target layer, $m$ refers to the number of risk evaluation grades, and $\gamma$ refers to the second-highest membership value in judgment matrix $B$ of the target layer.

The relationship between the $\alpha$ value and the effectiveness of the maximum membership principle is shown in Table 6 .

According to (30), the effective index $\alpha$ of this project is as follows:

$$
\alpha=\frac{5 \times 0.44-1}{2 \times 0.27 \times(5-1)}=0.56
$$

It can be seen from Table 6 that, since the effective index is $(0.5 \leq \alpha=0.56<1)$, the above-mentioned maximum membership principle is effective and can be used as the basis for the final risk evaluation.

\section{Countermeasures to Cope with Risks}

By analyzing the situation after the 100-ring tunneling test, the main problems figured out therefrom include inappropriate tunneling parameters, segment dislocation, segment damage, inadequate grouting, etc. This is in line with the above risk evaluation results. In addition, the setting range of the tunneling parameters and the treatment measures for related problems are proposed by analyzing the above-mentioned test data.

5.1. Test for Shield Tunneling Parameters. During the tunneling process of the test section, the shield tunneling parameters are tracked, including earth pressure, cutterhead torque and rotation speed, total thrust, tunneling speed, and excavation volume. Then, by analyzing the data of the aforementioned tunneling test, proper parameters are summarized to provide relevant experience for follow-up projects.

5.1.1. Total Thrust of Shield Machine. The total thrust changes of the shield machine are as shown in Figure 5. For the first 10 rings, it tunnels in the reinforced zone with the thrust controlled within $9000 \mathrm{kN}$. For the $11^{\text {th }} \sim 60^{\text {th }}$ rings, the tunneling thrust in fine-medium sand with mud is controlled within a range of $8000 \sim 15000 \mathrm{kN}$. For the $61^{\text {st }} \sim 73^{\text {rd }}$ rings, it tunnels in the zone that is composed of $1 \mathrm{~m}$ thick mucky soil at the bottom and fine-medium sand with
TABLE 6: The relationship between $\alpha$ value and the effectiveness of the maximum membership principle.

\begin{tabular}{lc}
\hline Interval & Effectiveness of maximum membership principle \\
\hline$\alpha \longrightarrow+\infty$ & Absolutely effective \\
$\alpha \in[1,+\infty)$ & Very effective \\
$\alpha \in[0.5,1)$ & Effective \\
$\alpha \in[0,0.5)$ & Slightly effective \\
$\alpha \longrightarrow 0$ & Ineffective \\
\hline
\end{tabular}

mud at the top, with the thrust controlled between 11000 and $14000 \mathrm{kN}$. For $74^{\text {th }} \sim 100^{\text {th }}$ rings, the shield machine tunnels in fine-medium sand with mud containing silt with thrust controlled between 10000 and $15000 \mathrm{kN}$. It shows comparatively stable thrust changes in general. But the attitude adjustment of the shield machine fluctuates a little bit during the tunneling process.

5.1.2. Torque and Rotation Speed of Cutterhead. The rotation speed of cutterhead runs at around $1 \mathrm{rpm}$ in the initial stage but is adjusted gradually to $0.9-1.3 \mathrm{rpm}$ when entering normal tunneling stage according to the tunneling speed. The torque is maintained steadily during the process. For the tunneling of the first 100 rings in the zone with a whole section of fine-medium sand with mud, the torque of the cutterhead is maintained between 500 and $1600 \mathrm{kN} \cdot \mathrm{m}$. For the changing process, please see Figure 6.

5.1.3. Tunneling Speed. The process of tunneling speed changes of the shield machine is as shown in Figure 7. It stands in stratum with whole section fine-medium sand with mud when the shield machine launches. For the first 1 5 rings, the speed is controlled within $40 \mathrm{~mm} / \mathrm{min}$. Then, the zone of the $6^{\text {th }} \sim 90^{\text {th }}$ rings still lies in stratum with whole section fine-medium sand with mud, so the speed is controlled between 30 and $60 \mathrm{~mm} / \mathrm{min}$. For the $80^{\text {th }} \sim 100^{\text {th }}$ rings, exceptional rolling angle parameter occurs, and the tunneling speed is reduced to below $30 \mathrm{~mm} / \mathrm{min}$ to correct the rolling angle.

5.1.4. Earth Pressure. For the tunnel interval after the shield machine finishes tunneling and moves out from the reinforced zone, the embedded depth is $9.74 \sim 13.24 \mathrm{~m}$, indicating that this interval is a shallow tunnel section. And its static earth pressure $P$ is as follows:

$$
\begin{aligned}
P & =k_{0} \gamma h \\
& =0.493 \times 18.9 \times(9.74 \sim 13.24) \\
& =90.8 \sim 123.4 \mathrm{kPa},
\end{aligned}
$$

where $k_{0}$ refers to the coefficient of static earth pressure, $h$ refers to the buried depth of tunnel (m), and $\gamma$ refers to bulk density of soil $\left(\mathrm{kN} / \mathrm{m}^{3}\right)$.

During the initial tunneling, the earth pressure is set strictly according to the theoretical earth pressure value but is adjusted and optimized according to the monitoring data of the surface settlement during the construction process. 


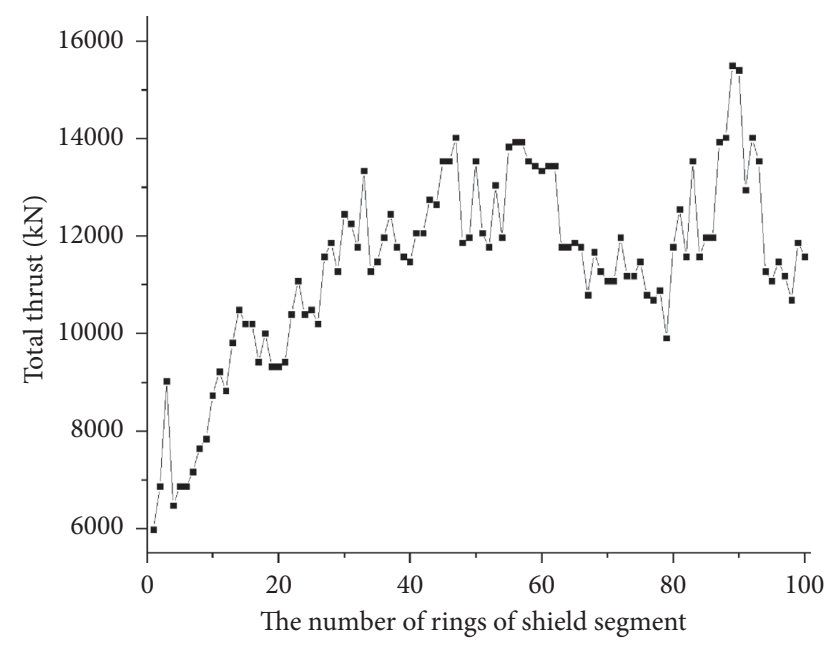

FIGURE 5: Variation law of total thrust of shield machine.

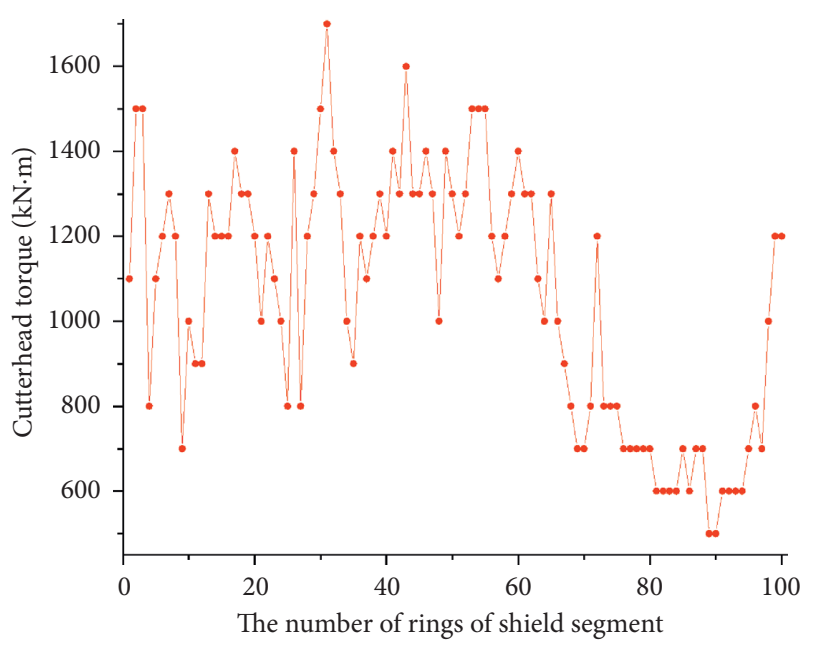

Figure 6: Variation law of torque of cutterhead.

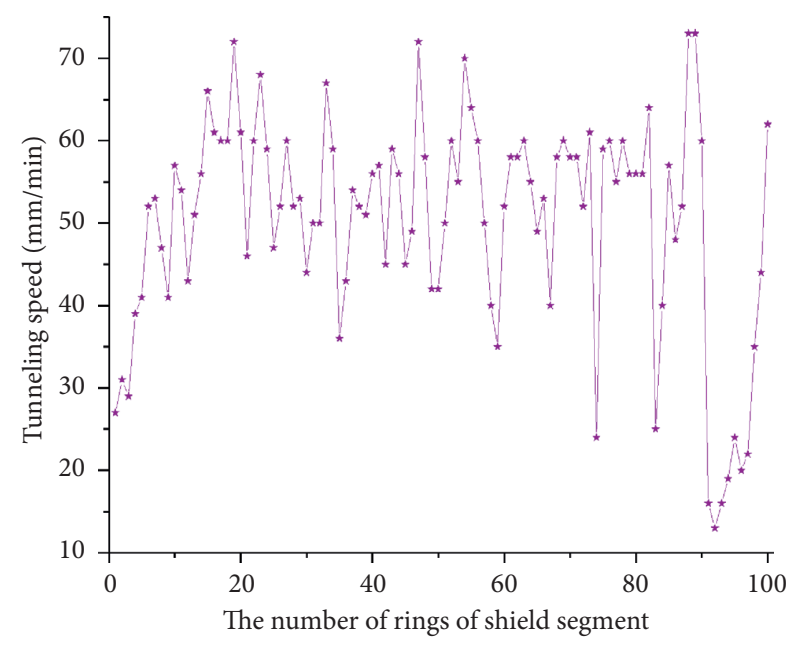

FIgURE 7: Variation law of tunneling speed.

Due to the significant settlement volume, the earth pressure slightly higher than the theoretical value is adopted in practical tunneling process, as shown in Figure 8.

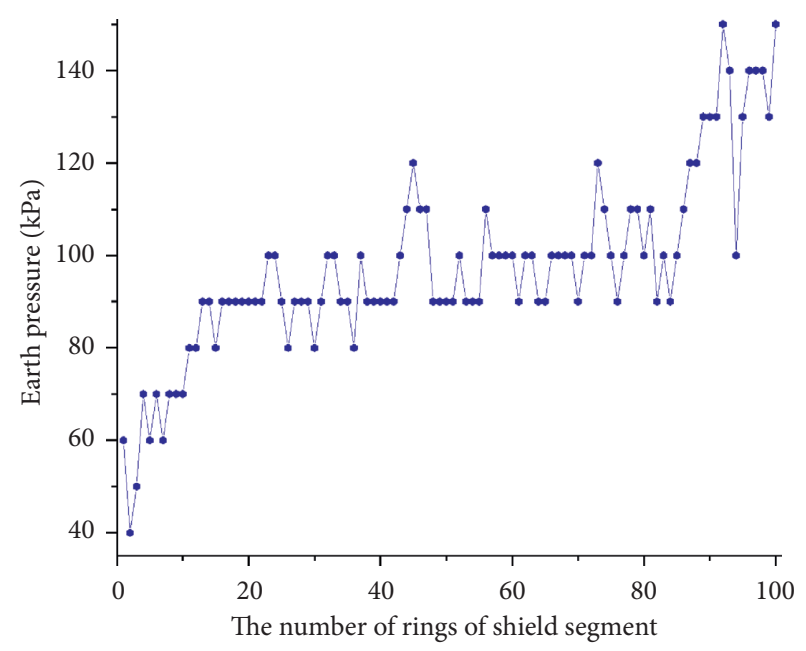

FIgURE 8: Variation law of earth pressure.

5.1.5. Excavation Volume. The theoretical excavation volume of each ring of segments is as follows:

$$
\begin{aligned}
Q_{1} & =\frac{n_{0} \pi D^{\prime 2} L}{4} \\
& =\frac{1.2 \times \pi \times 6.48^{2} \times 1.2}{4} \\
& =47.5 \mathrm{~m}^{3},
\end{aligned}
$$

where $n_{0}$ refers to the loose coefficient (1.2), $D^{\prime}$ refers to the cutterhead diameter $(6.48 \mathrm{~m})$, and $L$ refers to the segment width $(1.2 \mathrm{~m})$.

Therefore, the excavation volume of each ring of segment is about $47.5 \mathrm{~m}^{3}$. The amount of excavation produced by shield tunneling is controlled within $98 \% \sim 102 \%$, namely, within $46.5 \mathrm{~m}^{3} \sim 48.3 \mathrm{~m}^{3}$. According to the statistical analysis on the first 100 rings' excavation volumes (see Figure 9), it can be known that the mean value of the excavation volume of each ring is $45 \mathrm{~m}^{3}$, which is slightly lower than the theoretical value.

5.2. Synchronous Grouting Test of Shield Machine. Synchronous grouting is an important work step of shield construction. Full and even grouting can help to control surface settlement effectively.

The theoretical grouting quantity $Q$ is as follows:

$$
\begin{aligned}
Q & =V \cdot \lambda=\frac{\left(D^{\prime 2}-d^{2}\right) \cdot \pi \cdot L}{4} \\
& =\frac{\left(6.48^{2}-6.2^{2}\right) \times \pi \times 1.2}{4} \times(150 \% \sim 200 \%) \\
& =5.17 \sim 6.69 \mathrm{~m}^{3},
\end{aligned}
$$

where $V$ refers to the void caused by shield construction, $d$ refers to the outer diameter of the segment $(6.2 \mathrm{~m})$, and $\lambda$ is the grouting rate, which is taken as $150 \%-200 \%$ herein. 


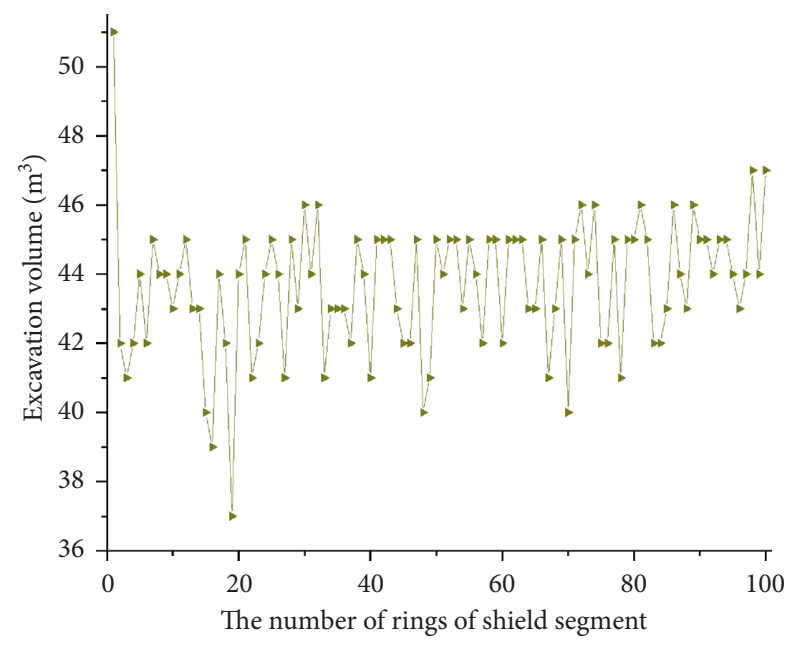

Figure 9: Variation law of excavation volume.

The synchronous grouting changes are as shown in Figure 10. The total grouting quantity is controlled between 4 and $7 \mathrm{~m}^{3}$.

\subsection{Segment Assembly Test}

5.3.1. Measures to Cope with Segment Dislocation. The changes of segment dislocation are as shown in Figures 11 and 12 . The segments have both circumferential and longitudinal dislocations in almost the same amounts. Most of the dislocations are $5 \mathrm{~mm}$. The largest circumferential dislocation happens in place between the $82^{\text {nd }}$ ring and the $83^{\text {rd }}$ ring, reaching a $9 \mathrm{~mm}$ dislocation quantity but smaller than the control quantity of $15 \mathrm{~mm}$. The largest longitudinal dislocation occurs in place between the L2 block and the $\mathrm{K}$ block of $86^{\text {th }}$ ring, showing an $18 \mathrm{~mm}$ dislocation quantity, which is greater than $10 \mathrm{~mm}$. Among the above, segments of a total of 4 rings (83/84/85/86) have longitudinal dislocation quantities exceeding the control quantity of $10 \mathrm{~mm}$ and need to be corrected and carried out with anti-crack and waterproof measures.

The measures to cope with the segment dislocation include the following:

(1) If possible, use the middle and upper grouting pipeline to conduct synchronous grouting.

(2) The sludge and sewage at the assembling part of the shield tail must be cleaned up before assembling the segments, and the sundries in the gap between the upstream surface of the segment of the previous ring and the shield tail must be cleaned up as well, thus to guarantee assembling the segments in a condition with no sundries or residual water.

(3) Bolt re-tightening must reach the design requirement, and the re-tightening measures shall be conducted at least three times (after assembling, during tunneling, and after moving out from the shield tail).

(4) The assembly of segments must follow the requirements of bottom-to-top sequence, left-and-right

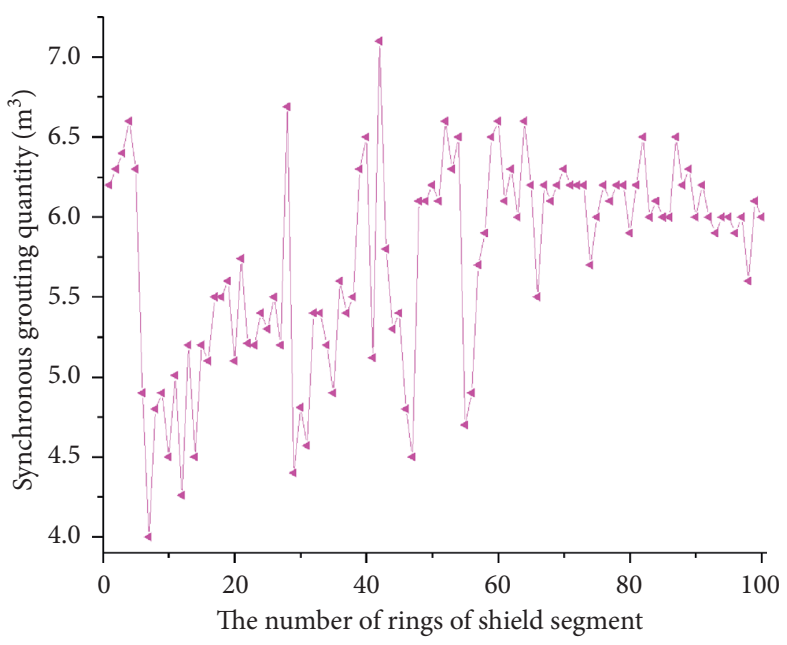

Figure 10: Variation law of synchronous grouting quantity.

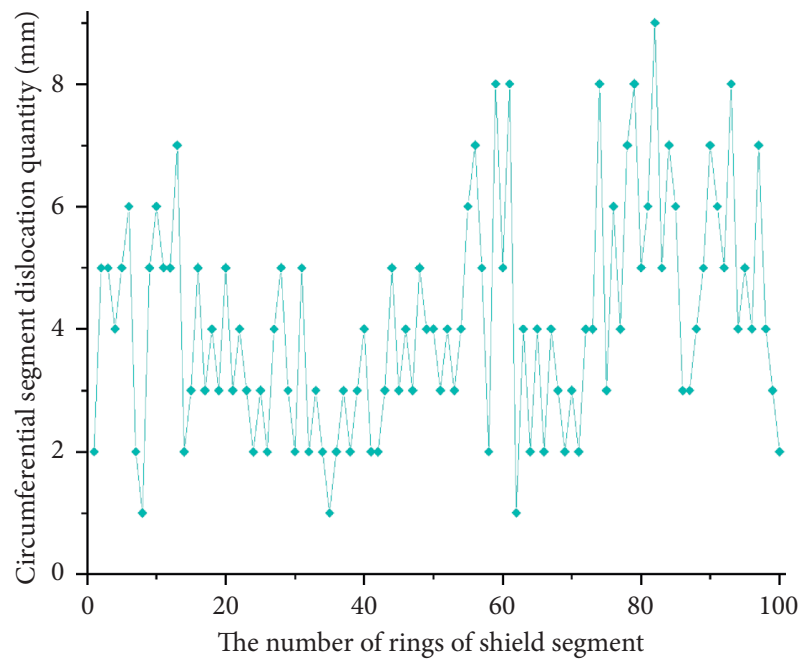

FIGURE 11: Variation law of circumferential segment dislocation quantity.

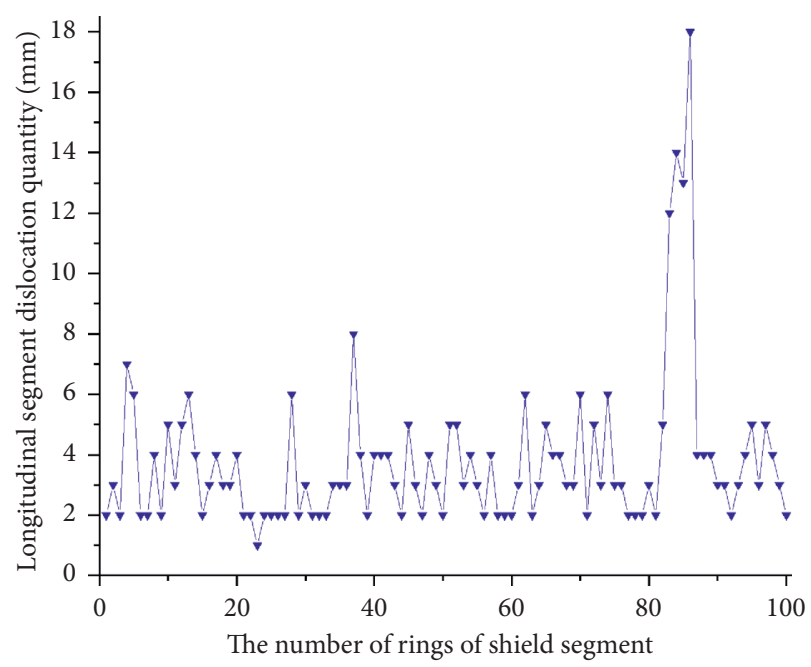

FIGURE 12: Variation law of longitudinal segment dislocation quantity. 
crossed arrangement, and sealing the top finally. Meanwhile, the fine-tuning device for segment installation must be used to adjust the inner arc between the to-be-installed segment and the installed segment into smooth connection status. The bolt holes shall be well aligned to make bolt insertion easy.

(5) Reasonably inject the shield tail grease to reduce the friction brought by the shield tail brush to the segment.

(6) Reasonably optimize the slurry proportion to reduce the uplifting volume of the segment.

5.3.2. Measures to Deal with Damaged Segment. On-site segment damage is shown in Figure 13. The obviously damaged segments include point 8 of block B2 of the $58^{\text {th }}$ ring, point 2 and point 12 of block B2 of the $56^{\text {th }}$ ring, point 12 of block B1 of the $52^{\text {nd }}$ ring, and point 11 of block K of the $53^{\text {rd }}$ ring.

The fixed segment is as shown in Figure 14. The measures to deal with damaged segment are as follows:

(1) Strengthen the acceptance inspection of segments. It is strictly forbidden to transport segments with uneven surfaces, missing edges or corners, excessive air bubbles, pitted surfaces, and exposed rebar to the construction site.

(2) Strengthen the training to the assembly workers, make clear assembly identification for each ring, and assign special personnel to take charge of it.

(3) During the assembling of the segments, in any case that the uneven local ring surface is found, the assembly shall be paused to adjust the force transmission liner timely, to guarantee the flatness of the ring surface.

(4) Conduct grouting strictly according to the designed synchronous grouting quantity.

(5) Strictly implement the bolt tightening regulations during the tunneling process, thus to prevent the segment floating that may result in segment dislocation and damage.

(6) Fix the segments strictly according to the segment fixation plan, and adjust the color difference properly.

(7) Maintain the shield posture and the clearance between the shield tails, and clean up the mud in the shield tails in time.

5.3.3. Measures to Deal with Segment Attitude Deviation. The changes of on-site segment attitude deviation are as shown in Figures 15 and 16. The segment suffers not only the horizontal deviation but also the vertical deviation. And the vertical deviation is greater than the horizontal deviation. The segments deviate upward in the fine-medium sand with mud. The largest vertical deviation happens at the $45^{\text {th }}$ ring, which is $78.1 \mathrm{~mm}$ deviation quantity. At the horizontal

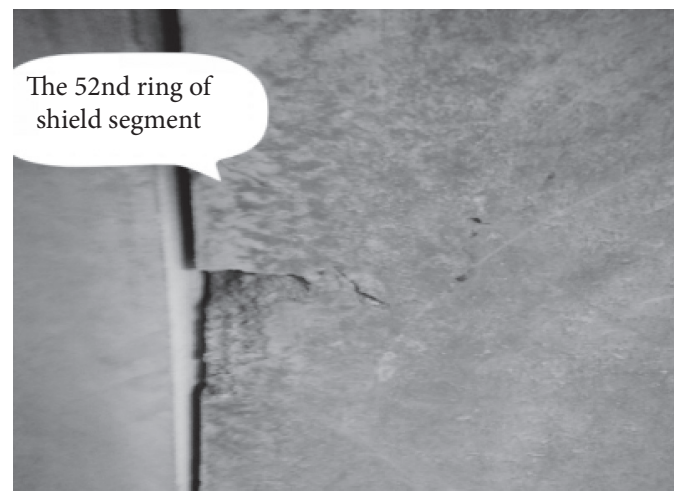

FIGURE 13: On-site segment damage.

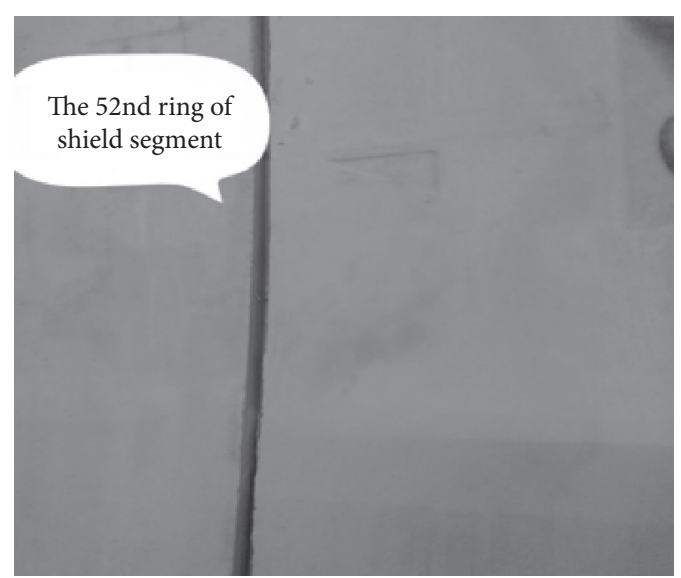

FIgURE 14: Segment repair.

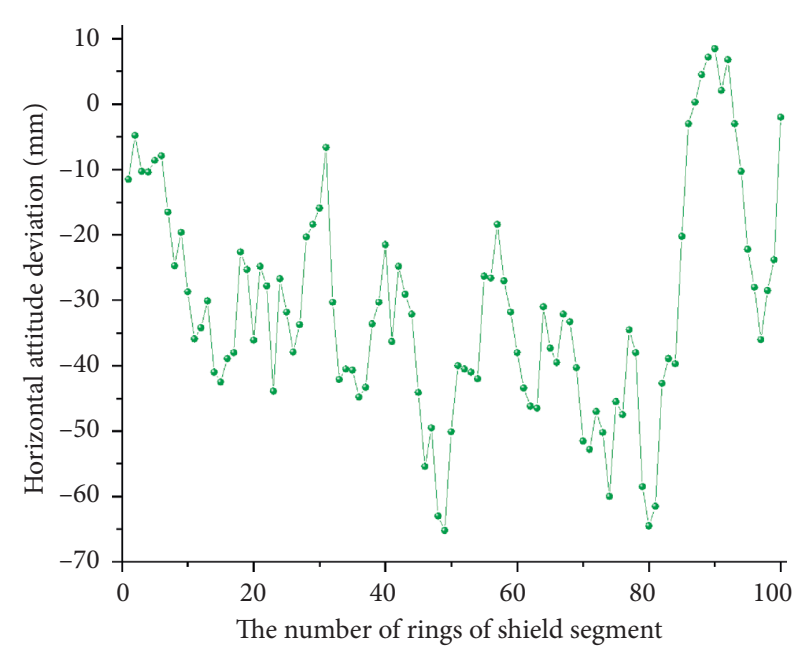

FIgURE 15: Variation law of horizontal attitude deviation of segments.

direction, rightward deviation of segments happens, for which the largest deviation occurs at the $49^{\text {th }}$ ring, reaching $65.2 \mathrm{~mm}$.

The measures to control segment deviation include the following: 


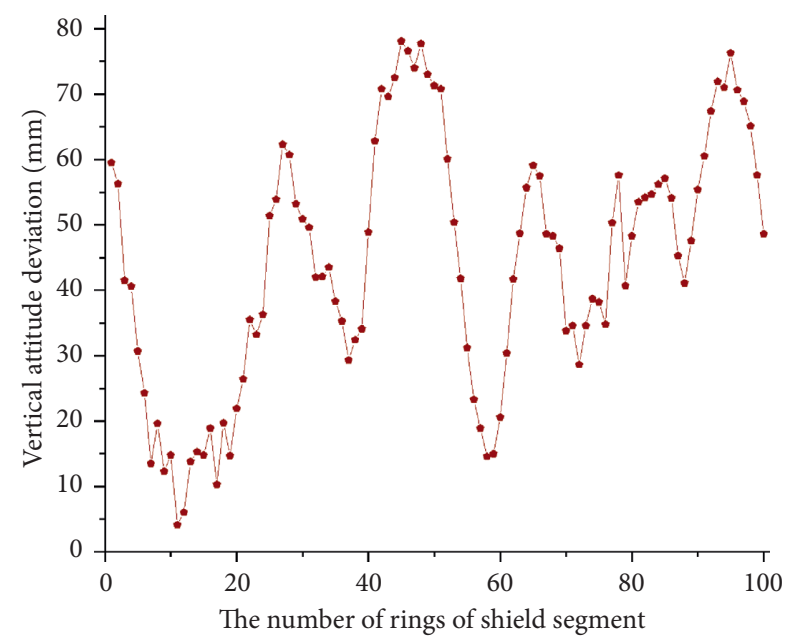

FIGURE 16: Variation law of horizontal attitude deviation of segments.

(1) For the tunneling in the fine-medium sand with mud, the measure of controlling tunneling speed should be taken. Each work shift shall tunnel for 6-7 rings in their shift and follow up the secondary double liquid grouting timely. Currently, the method of grouting once every two rings is applied on-site.

(2) Targeting the problems of insufficient shield machine thrust and hard-to-control attitude, the method of enlarging soil pressure properly is adopted to control the uplifting problem.

(3) Adjust the proportion of synchronous grouting to shorten the initial setting time. The initial setting time of the current synchronous grouting is 6-7 hours, and the problem of segment uplifting has been improved to a certain extent.

(4) When the shield machine tunneling attitude is well controlled, it shall adjust the vertical trend of the shield machine slowly, to guarantee that the formed segments meet the design requirements.

\section{In Situ Monitoring}

Figure 17 shows how surface settlement changes, by 7 April 2018, as the right tunneling line of the metro section between Binhai New Town Station and Lianhua Station is under construction.

As the shield machine is processing, the surface settlement is on the rise. The cumulative subsidence is $-104.04 \mathrm{~mm}$ at the most, exceeding the maximum subsidence control of urban road, $-30 \mathrm{~mm}$. Additionally, daily subsidence peaks at $-26.12 \mathrm{~mm}$. The large settlement will not give rise to safety problems during the construction of this metro section which is mainly covered by farms and fishponds, whereas it can bring about heavy economic losses. Therefore, surface settlement needs to be controlled by modifying tunneling parameters.

After relevant tests, the tunneling parameters are modified. Total thrust power is $8000-16000 \mathrm{kN}$. The

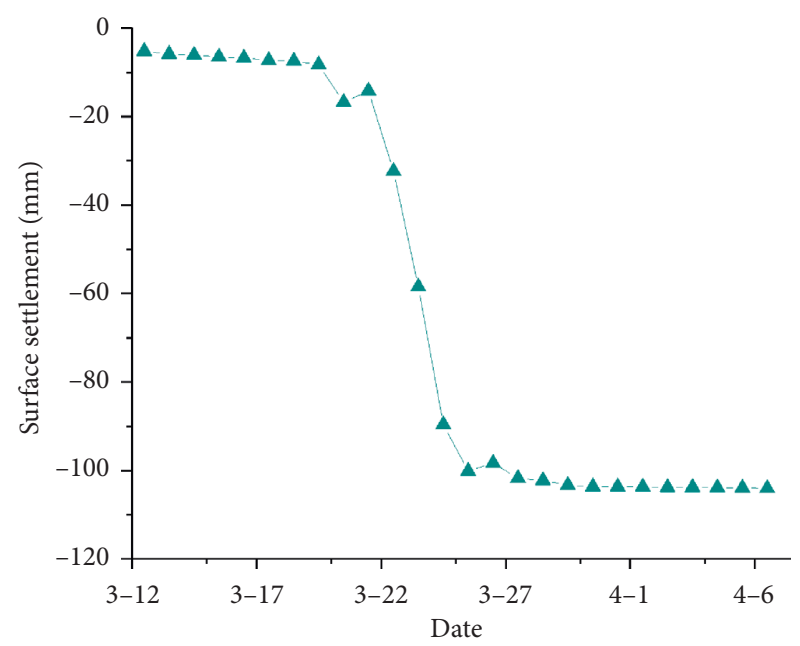

FIGURE 17: Changes in surface settlement.

cutterhead torque is $1000-1600 \mathrm{kN} \cdot \mathrm{m}$, and its rotating speed is around $1.0 \mathrm{rpm}$. The thrust speed is about $50 \mathrm{~mm} / \mathrm{min}$. Earth pressure lies between 120 and $140 \mathrm{kPa}$, and the volume of excavation is tested as $45 \mathrm{~m}^{3}$. The amount of synchronous grouting is $5-7 \mathrm{~m}^{3}$. When these modified tunneling parameters are applied, the surface settlement is developing in a steady way.

\section{Conclusions}

(1) After analyzing the construction features of modified shield machines applicable in soft soils, it is concluded that relevant risks exist in operating steps including shield launching, receiving, and tunneling, segment assembly, and grouting, as well as in the surroundings. The risks of shield tunneling, segment assembly, and grouting rank high indicate that prepreparations are needed to counteract emergencies during construction.

(2) A risk evaluation model is specifically established for the modified shield tunneling in soft soils. This model weighs risk factors through triangular fuzzy numbers, which helps avoid subjectivity and better reflects the fuzziness of risk factors, without the consistency check as analytic hierarchy process (AHP) does. Furthermore, efficacy indices are used to ensure the reliability of maximum membership principle, rendering the fuzzy comprehensive evaluation method more accurate.

(3) This specific model is applied to the construction of one section tunnel (Binhai New Town-Lianhua) of Metro Line 6 in Fuzhou City. The model rates this project as high-risk. High risks exist in shield tunneling, segment assembly, and grouting. The same assessment is shown by data obtained from tunneling tests on-site and surface settlement measurement, which again certifies the reliability of this model.

(4) Recommended tunneling parameters and countermeasures against segment dislocation, damage, and 
attitude deviation can be derived from data collected from field tests of 100-ring tunneling. It is hoped that these findings will serve as a reference for future application of modified shield tunneling in soft soils.

\section{Data Availability}

The data used to support the findings of this study are included within the article.

\section{Conflicts of Interest}

The authors declare that there are no conflicts of interest regarding the publication of this paper.

\section{Acknowledgments}

This work was funded by the China Power Construction Major Project (DJ-ZDXM-2017-23).

\section{References}

[1] R. Zumsteg and L. Langmaack, "Mechanized tunneling in soft soils: choice of excavation mode and application of soilconditioning additives in glacial deposits," Engineering, vol. 3, no. 6, pp. 863-870, 2017.

[2] K. Cui and W. Lin, "Muck problems in subway shield tunneling in sandy cobble stratum," Polish Maritime Research, vol. 23, no. s1, pp. 175-179, 2016.

[3] N. Bilgin, H. Copur, and C. Balci, "Effect of replacing disc cutters with chisel tools on performance of a TBM in difficult ground conditions," Tunnelling and Underground Space Technology, vol. 27, no. 1, pp. 41-51, 2012.

[4] S. Cao, J. Cui, Y. Fang, and R. Deng, "Performance of slurry tbm tunnelling in sandy cobble ground-a case study in Lanzhou," KSCE Journal of Civil Engineering, vol. 23, no. 7, pp. 3207-3217, 2019.

[5] Q. M. Gong, L. J. Yin, and Q. R. She, "TBM tunneling in marble rock masses with high in situ stress and large groundwater inflow: a case study in China," Bulletin of Engineering Geology and the Environment, vol. 72, no. 2, pp. 163-172, 2013.

[6] J. Zhao, Q. M. Gong, and Z. Eisensten, "Tunnelling through a frequently changing and mixed ground: a case history in Singapore," Tunnelling and Underground Space Technology, vol. 22, no. 4, pp. 388-400, 2007.

[7] J. Tebar and N. Alonso, "Quejigares tunnels. Excavation in soft ground and rock with a mixed shield EPB," in Proceedings of the World Tunnel Congress: Underground-the Way to the Future, WTC 2013, Taylor and Francis-Balkema, Geneva, Switzerland, pp. 2046-2053, May 2013.

[8] K. Rafie, S. Skelhorn, and A. Mitchell, "EPB tunnelling at high pressures: customization of tunnelling systems for Port Mann tunnel," in Proceedings of the 2013 Rapid Excavation and Tunneling Conference, RETC 2013, Society for Mining, Metallurgy and Exploration, Washington, DC, USA, pp. 967-986, June 2013.

[9] X. Li and D. Yuan, "Creating a working space for modifying and maintaining the cutterhead of a large-diameter slurry shield: a case study of Beijing railway tunnel construction," Tunnelling and Underground Space Technology, vol. 72, pp. 73-83, 2018.
[10] N. Zhang, J. S. Shen, A. Zhou, and A. Arulrajah, "Tunneling induced geohazards in mylonitic rock faults with rich groundwater: a case study in Guangzhou," Tunnelling and Underground Space Technology, vol. 74, pp. 262-272, 2018.

[11] H. Pan, J. Gou, Z. Wan et al., "Research on coupling degree model of safety risk system for tunnel construction in subway shield zone," Mathematical Problems in Engineering, vol. 2019, Article ID 5783938, 19 pages, 2019.

[12] R. L. Sousa and H. H. Einstein, "Risk analysis during tunnel construction using Bayesian networks: Porto Metro case study," Tunnelling and Underground Space Technology, vol. 27, no. 1, pp. 86-100, 2012.

[13] X. L. Jia, Q. M. Dai, and H. Z. Yang, "Susceptibility zoning of karst geological hazards using machine learning and cloud model," Cluster Computing, vol. 22, pp. S8051-S8058, 2019.

[14] X. H. Deng, T. Xu, and R. Wang, "Risk evaluation model of highway tunnel portal construction based on BP fuzzy neural network," Computational Intelligence and Neuroscience, vol. 2018, Article ID 8547313, 16 pages, 2018.

[15] A. Pourtaghi and M. A. Lotfollahi-Yaghin, "Wavenet ability assessment in comparison to ANN for predicting the maximum surface settlement caused by tunneling," Tunnelling and Underground Space Technology, vol. 28, pp. 257-271, 2012.

[16] W. Zhang, K. Sun, C. Lei, Y. Zhang, H. Li, and B. F. Spencer, "Fuzzy analytic hierarchy process synthetic evaluation models for the health monitoring of shield tunnels," Computer-Aided Civil and Infrastructure Engineering, vol. 29, no. 9, pp. 676-688, 2014.

[17] F. Barpi and D. Peila, "Influence of the tunnel shape on shotcrete lining stresses," Computer-Aided Civil and Infrastructure Engineering, vol. 27, no. 4, pp. 260-275, 2012.

[18] K.-C. Hyun, S. Min, H. Choi, J. Park, and I.-M. Lee, "Risk analysis using fault-tree analysis (FTA) and analytic hierarchy process (AHP) applicable to shield TBM tunnels," Tunnelling and Underground Space Technology, vol. 49, pp. 121-129, 2015.

[19] Y. Cao and S. Xiong, "A sustainable financing credit rating model for China's small- and medium-sized enterprises," Mathematical Problems in Engineering, vol. 2014, Article ID 861085, 5 pages, 2014.

[20] M. Jakšić, S. Moljević, A. Aleksić et al., "Fuzzy approach in ranking of banks according to financial performances," Mathematical Problems in Engineering, vol. 2016, Article ID 6169586, 11 pages, 2016.

[21] F.-Y. Hsiao, S.-H. Wang, W.-C. Wang, C.-P. Wen, and W.-D. Yu, "Neuro-fuzzy cost estimation model enhanced by fast messy genetic algorithms for semiconductor hookup construction," Computer-Aided Civil and Infrastructure Engineering, vol. 27, no. 10, pp. 764-781, 2012.

[22] W.-C. Huang, C.-H. Jhong, and J.-F. Ding, "Key factors influencing sustainable development of a green energy industry in Taiwan," Mathematical Problems in Engineering, vol. 2013, Article ID 791896, 10 pages, 2013.

[23] Y. Li, Y. Li, Z. Han, G. Li, K. Gao, and Z. Wang, "Determination of optimal opening scheme for electromagnetic loop networks based on fuzzy analytic hierarchy process," Mathematical Problems in Engineering, vol. 2016, Article ID 3965608, 11 pages, 2016.

[24] W. Graf, S. Freitag, J.-U. Sickert, and M. Kaliske, "Structural analysis with fuzzy data and neural network based material description," Computer-Aided Civil and Infrastructure Engineering, vol. 27, no. 9, pp. 640-654, 2012.

[25] H. Li, W.-J. Yi, and X.-X. Yuan, "Fuzzy-valued intensity measures for near-fault pulse-like ground motions," Computer-Aided Civil and Infrastructure Engineering, vol. 28, no. 10, pp. 780-795, 2013. 
[26] W. B. Tagherouit, S. Bennis, and J. Bengassem, "A fuzzy expert system for prioritizing rehabilitation of sewer networks," Computer-Aided Civil and Infrastructure Engineering, vol. 26, no. 2, pp. 146-152, 2011.

[27] A. Bianchini, "Fuzzy representation of pavement condition for efficient pavement management," Computer-Aided Civil and Infrastructure Engineering, vol. 27, no. 8, pp. 608-619, 2012.

[28] M. Dell'Orco and M. Mellano, "A new user-oriented index, based on a fuzzy inference system, for quality evaluation of rural roads," Computer-Aided Civil and Infrastructure Engineering, vol. 28, no. 8, pp. 635-647, 2013.

[29] M. B. Anoop, B. K. Raghuprasad, and K. Balaji Rao, “A refined methodology for durability-based service life estimation of reinforced concrete structural elements considering fuzzy and random uncertainties," Computer-Aided Civil and Infrastructure Engineering, vol. 27, no. 3, pp. 170-186, 2012.

[30] G. Zhang, C. Wang, Y. Jiao et al., "Collapse risk analysis of deep foundation pits in metro stations using a fuzzy Bayesian network and a fuzzy AHP," Mathematical Problems in Engineering, vol. 2020, Article ID 4214379, 18 pages, 2020.

[31] H. M. Lyu, W. J. Sun, S. L. Shen et al., "Risk assessment using a new consulting process in fuzzy AHP," Journal of Construction Engineering and Management, vol. 146, no. 3, p. 12, 2020.

[32] Y. Wang, B. Liu, and Y. Qi, “A risk evaluation method with an improved scale for tunnel engineering," Arabian Journal for Science and Engineering, vol. 43, no. 4, pp. 2053-2067, 2017.

[33] T. L. Saaty, "How to make a decision: the analytic hierarchy process," European Journal of Operational Research, vol. 48, no. 1, pp. 9-26, 1990.

[34] B. Wang, C. Mo, C. He et al., "Fuzzy synthetic evaluation of the long-term health of tunnel structures," Applied Sciences, vol. 7, no. 2, 2017.

[35] J. Khademi Hamidi, K. Shahriar, B. Rezai, J. Rostami, and H. Bejari, "Risk assessment based selection of rock TBM for adverse geological conditions using fuzzy-AHP," Bulletin of Engineering Geology and the Environment, vol. 69, no. 4, pp. 523-532, 2010.

[36] A. Yazdani-Chamzini and S. H. Yakhchali, "Tunnel boring machine (TBM) selection using fuzzy multicriteria decision making methods," Tunnelling and Underground Space Technology, vol. 30, pp. 194-204, 2012.

[37] X. Wang, K. Shi, Q. Shi et al., "A normal cloud model-based method for risk assessment of water inrush and its application in a super-long tunnel constructed by a tunnel boring machine in the arid area of northwest China," Water, vol. 12, no. 3, p. 644, 2020.

[38] A. Aalianvari, H. Katibeh, and M. Sharifzadeh, "Application of fuzzy Delphi AHP method for the estimation and classification of Ghomrud tunnel from groundwater flow hazard," Arabian Journal of Geosciences, vol. 5, no. 2, pp. 275-284, 2012.

[39] H. Yao, H.-B. Zhou, L.-B. Cai, and Y.-F. Zhang, "Fuzzy synthetic evaluation on construction risk of EPBS during tunnelling in soft soil area," Yantu Lixue/Rock and Soil Mechanics, vol. 28, no. 8, pp. 1753-1756, 2007.

[40] L. Han, Q. Mei, Y.-m. Lu et al., "Analysis and study on AHPfuzzy comprehensive evaluation," China Safety Science Journal, vol. 14, no. 7, pp. 89-92, 2004. 